\title{
与时俱进地做好化学实验教学实验室的建设与管理
}

翁玉华，许振玲，潘荵，张春艳，吕银云，阮婵姿，董志强，阮永红，张来英，方雪明， 彭淑女, 任艳平 ${ }^{*}$

厦门大学化学化工学院, 化学国家级实验教学示范中心(厦门大学), 福建 厦门 361005

摘要：概括总结厦门大学化学国家级实验教学示范中心在安全、规范、高效服务于实验教学的实验室细化建设与管 理理念指导下的实验室建设与管理的总体思想, 融管理于建设中、寓规范化管理于人性化服务的 “以学生为中心” 的实验室细化建设与管理的 “中心标准” , 以及在实验教学及其管理、实验仪器及化学试剂管理的信息化和智能化 建设方面所取得的进展等, 以有利于其他高校在更高起点、更高层次和更高目标上推进实验室细化建设与管理工作。

关键词：细化建设与管理; 教学实验室; 实验教学示范中心标准; 示范与辐射效应

中图分类号: G64; 06

\section{Keep Pace with the Times to Do a Good Job in the Construction and Management of Experimental Chemistry Teaching Laboratory}

Yuhua Weng, Zhenling Xu, Rui Pan, Chunyan Zhang, Yinyun Lü, Chanzi Ruan, Zhiqiang Dong, Yonghong Ruan, Laiying Zhang, Xueming Fang, Shunü Peng, Yanping Ren *

National Demonstration Center for Experimental Chemistry Education (Xiamen University), College of Chemistry and Chemical Engineering, Xiamen University, Xiamen 361005, Fujian Province, China.

\begin{abstract}
This article mainly summarizes the overall ideas of laboratory construction and management based on the concept of laboratory refinement construction and management that helps conduct laboratory teaching safely and efficiently and in compliance with standards at the National Experimental Teaching Demonstration Center of Chemistry, Xiamen University. The university combines standardized management with humanized service, as well as the progress of informationization and intelligent construction of laboratory teaching and its management, and management of experimental instruments and chemical reagents. These summaries will help other universities to promote laboratory construction and management at a higher starting point, a higher level and a higher goal.
\end{abstract}

Key Words: Refine construction and management; Chemistry teaching laboratory;

Laboratory center standards; Demonstration and radiation effects

厦门大学化学实验教学中心成立于2000年, 2006年被教育部批准为首批 “国家级实验教学示范 中心”, 在 20 年的建设实践和发展过程中, 且行、且思、且悟、且改、且进, 如今在化学实验教学的 “硬件”和 “软件”建设方面都取得了显著成效, 要用好这 “硬件”和 “软件”资源, 已步入以提 高实验教学质量为核心、以培养拔尖创新人才为目标的内涵式发展阶段。

所谓内涵建设就是要以 “学生为中心”, 紧紧围绕着学校双一流建设和人才培养目标, 要有 “与

收稿: 2021-09-24; 录用: 2021-11-15; 网络发表: 2022-03-04

“通讯作者, Email: ypren@xmu.edu.cn

基金资助: 2021年度教育部 “基础学科拔尖学生培养计划2.0” 研究课题(20212058); 厦门大学2021年本科课程思政教学研究项目; 厦门大学教 学改革研究项目(教材研究专项, JG20210904); 首批国家级线下一流课程建设项目 
时俱进” 的理念, 力求创新性地把我们实验教学的各方面工作以及实验室的建设与管理工作做细、 做实、做精。前文 ${ }^{[1-18]}$ 已经报道了厦门大学化学国家级实验教学示范中心(下简称 “厦大实验中心”) 多年来围绕着内涵建设在实验教学以及实验室建设与管理方面做细、做实的一些典型实例, 如在实 验室安全、环境、仪器、试剂的细化管理和实验过程管理以及实验细化准备等方面的一些具体做法、 体会和经验。为了进一步有效推广国家级实验教学示范中心的先进经验, 扩大受益面, 充分发挥示 范辐射作用, 本文主要概括总结近几年来 “厦大实验中心” 在认识实验室建设与管理的重要性与必 要性的基础上, 在其率先提出的安全、规范、高效服务于实验教学的实验室细化建设与管理理念指 导下的实验室建设与管理的总体思想, 融管理于建设中、寓规范化管理于人性化服务的 “以学生为 中心” 的实验室细化建设与管理的 “中心标准” 等, 以有利于其他高校在更高起点、更高层次和更 高目标上推进实验室细化建设与管理工作。

\section{1 做好化学实验教学实验室建设与管理的重要性和必要性}

化学实验教学实验室作为化学实验教学的重要依托, 高水平的实验室建设与管理兼具高效服务 于实验教学的专业功能和润物无声的育人功能, 对培养学生的专业兴趣和专业素养、环保意识以及 责任意识都具有重要作用 ${ }^{[18]}$

\section{1 做好化学实验教学实验室建设与管理的重要性}

实验室建设与管理是教辅人员的工作, 但从图1可以清楚地看出实验室建设与管理效果对人才 培养有很大影响。因为实验室里一切 “静” 和 “动” (实验安全、实验环境、实验条件、教学内容、 教师水平、教师责任心等)都对学生产生有形、无形的影响和教育, 良好的实验环境吸引学生在实验 室安心研究和探索, 有利于培养学生的科学精神和安全、环保意识及责任心等, 我们可以看到实验 室是一个全方位的育人环境, 也是 “课程思政” 的情境场所。所以, 在实验室里的一切都要做到高 标准、高要求……, 尽管学生可能还一时达不到我们的要求和目标, 但让学生看到和感受到什么是 真正的好, 并朝此方向努力。

\begin{tabular}{|c|c|c|c|}
\hline 实验室里一切 “静” 和 “动” & 有形、无形教育 & & 防护意识 \\
\hline 实验安全 & 培养学生安全、环保意识 & - & 行动措施 \\
\hline 实验环境 & 水、火、电、毒、伤 & & 责任意识 \\
\hline 实验条件 & 吸引学生研究和探索 & & \\
\hline 教学内容等 & 培养学生科学精神 & & 高标准 \\
\hline 教师水平 & 培养学生责任心 & & 高要求 \\
\hline 教师责任心等 & 看到、感受到真 “好” & 努力方向 & 高目标 \\
\hline \multicolumn{2}{|c|}{ 实验室是一个全方位的育人环境 } & & 高水平 \\
\hline \multicolumn{2}{|c|}{ 实验室是 “课程思政” “情境” 场所 } & & 高效率 \\
\hline
\end{tabular}

图1＼cjkstart实验室建设和管理与人才培养关系内容概要

\section{2 做好化学实验教学实验室建设与管理的必要性}

目前, “厦大实验中心” 每学年承担全校 10 个学院 3600 多名本科生的 $26 门$ 门次实验课, 需要以化 院为主全校招聘的医学、公卫、能源等学院具有化学背景的指导教师 140 人次, 还有来自不同学科 专业、研究方向的110名硕士和博士研究生作为助教参与实验教学, 同时还承担科研服务、中学生的 奥赛培训、中小学生的科普宣传教育以及研学活动等工作。所以, “厦大实验中心” 实验室是来自校 内外不同认知层次的师生高频度交流的重要场所, 影响面广、影响力大。 
面对上述情形, 促使我们要思考的问题是如何与时俱进地精准做好实验室建设与管理? 如何高 效服务于实验教学? 如何做到 “以学生为中心” 的人性化服务? 如何提高教辅人员的工作效能? 下 面就概括总结几年来 “厦大实验中心”面对上述问题所进行的一些探索和实践经验 ${ }^{[1-18]}$ 。

\section{2 实验室细化建设与管理的总体思路}

“厦大实验中心” 实验室建设与管理的总体思路如图2。面对中国高校在教学实验室建设与管理 方面普遍存在重建设、轻管理现象, “厦大实验中心” 在其率先提出安全、规范、高效服务实验教 学的实验室建设与管理理念指导下, 本着 “厦大实验中心” 实验室全局设计、局部建设、分步实施 的建设思路 ${ }^{[12]}$, 在整修或新建实验室整体设计和建设实施过程中就融入管理理念, 即在新建或整修 改造时就要考虑管理及效果, 强调建设与管理要协同发展, 注重建设与管理的 “内涵”, 一边建设, 一边管理实践应用, 一边建设改进，寓管理于建设中，打破实验室建设和管理 “两张皮” 的局面， 与时俱进地将实验室建设与管理工作做细、做实、做精, 全力服务实验教学。

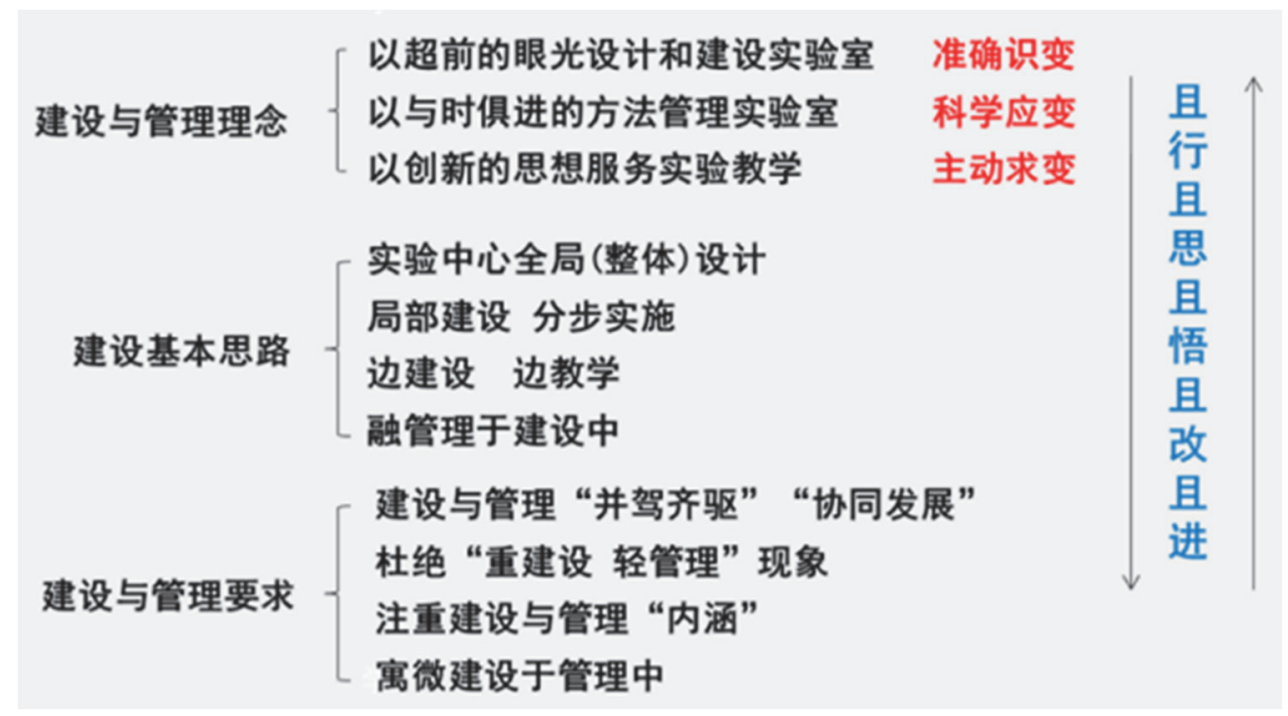

图2“厦大实验中心”实验室建设与管理的总体思路概要

\section{3 “以学生为中心” 的实验室细化建设与管理的 “中心标准”}

要呈现良好的实验室建设与管理效果, 要在精准设计、精准建设的基础上做到精准管理, 要在 管理实践过程中融入微建设。“厦大实验中心”从实验室建设与管理的细微做起, 主要从安全、环境、 仪器、试剂管理等几个方面 “以学生为中心” 与时俱进地实行 “科学化” 的细化管理和 “人性化” 的细化管理, 并在建设与管理实践的基础上, 摸索总结出一套完善的、行之有效的 “以学生为中心” 的 “科学化” 和 “人性化” 的实验室细化建设与管理 “中心标准”, 其内容概要如图3所示。

关于图3中 “科学化” 和 “人性化” 的实验室细化建设与管理 “中心标准” 中对实验楼道和实验 室的消防、急救设施及用品、公用实验物品以及普通仪器等摆放遵循 “三定” 原则的具体实施情况 及效果见文献 ${ }^{[11-14]}$ 。下面概括介绍有关 “中心标准” 中对实验室精密仪器和学生实验室化学试剂的 管理实施情况。

\section{1 精密仪器的 “五防”、标识 “四明” 和 “三位一体” 管理}

“厦大实验中心” 的精密仪器通常摆放在仪器室的固定位置, 并对仪器设备进行防尘、防潮、 防腐、防震、防扰 “五防” 管理和仪器名称明确、型号明确、性能明确、状态明确 “四明” 管理, 每台仪器均配有 “仪器使用登记本”, 要求学生用完仪器要进行清理和检查仪器状态并填写 “仪器使 


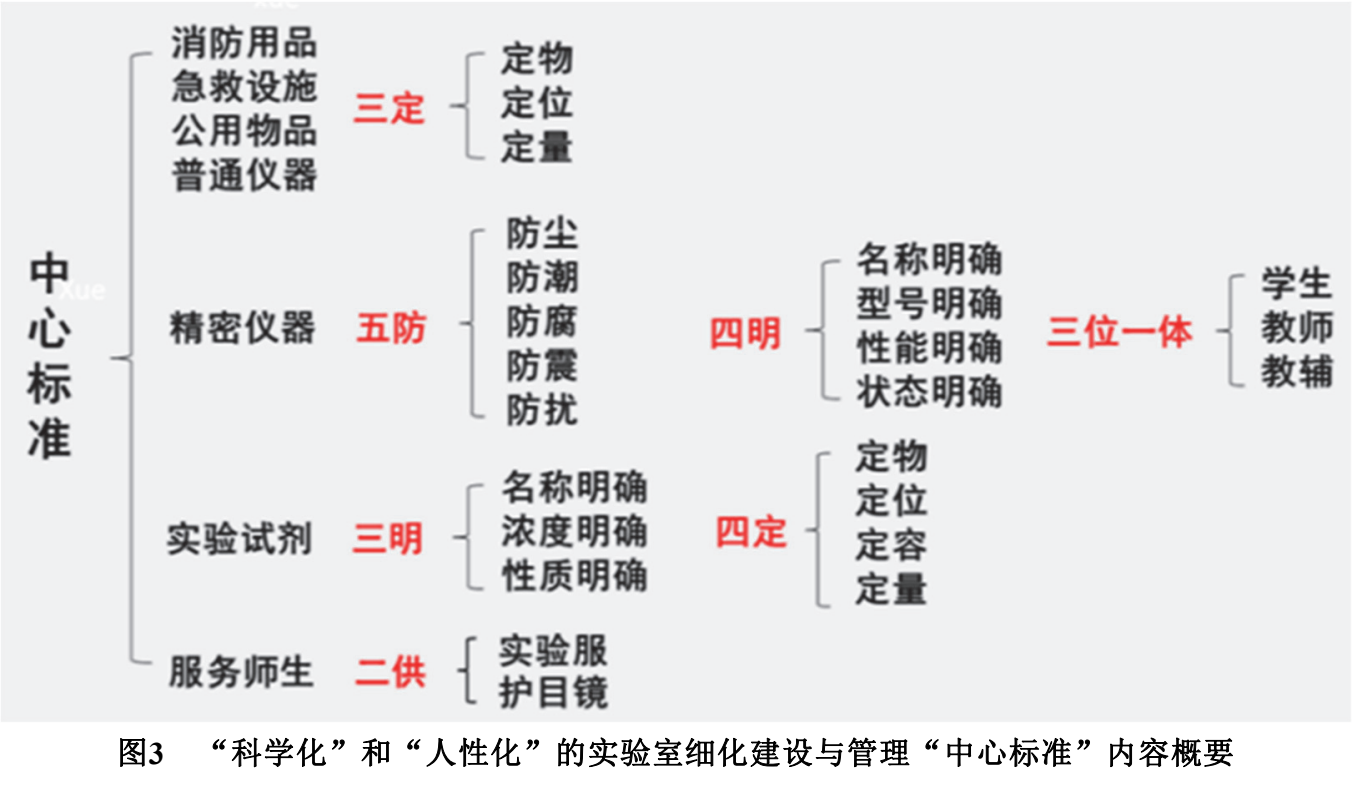

用登记本”, 并由指导教师检查和签名确认, 再由教辅进行最后把关, 即实行的是学生、教师、教辅 “三位一体” 的三级管理。通过 “五防” “四明” 和 “三位一体” 管理(图4)。不仅为学生获得科学准 确的实验数据提供了保障; 同时也有利于提高实验教学效率以及教辅人员的工作效率; 也延长了仪 器的使用寿命，节约了经费(节约即环保)等。

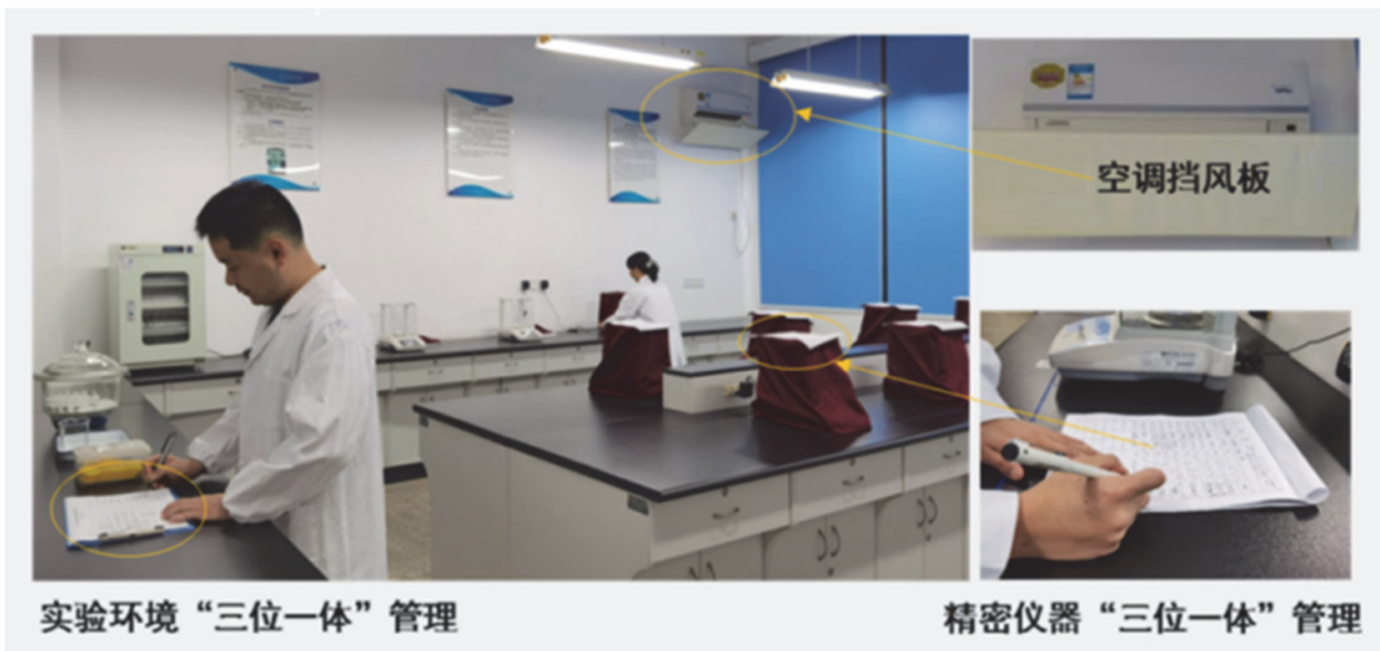

图4 精密仪器的 “五防”、标识 “四明” 和 “三位一体” 管理示例

\section{2 学生实验室化学试剂实行 “标识三明” 和 “四定” 管理}

为了让学生对化学试剂进行科学认识及安全、快速、准确取用, “厦大实验中心” 对学生实验中 所使用的普通化学试剂或易燃、易爆、强腐蚀性及易挥发性化学试剂实行名称明确(什么试剂)、浓度 明确(多大浓度)、性质明确(毒害性) “标识二明” 或 “标识三明” 和定物、定位、定容、定量(即什么 试剂放在哪儿、多大体积瓶装、放几瓶) “四定” 管理, 如图5所示。通过对学生实验室化学试剂实行 “标识二明” 或 “标识三明” 和 “四定” 管理, 不仅提高了学生的实验效率, 也有利于培养学生安 全使用试剂的意识及环保意识。 


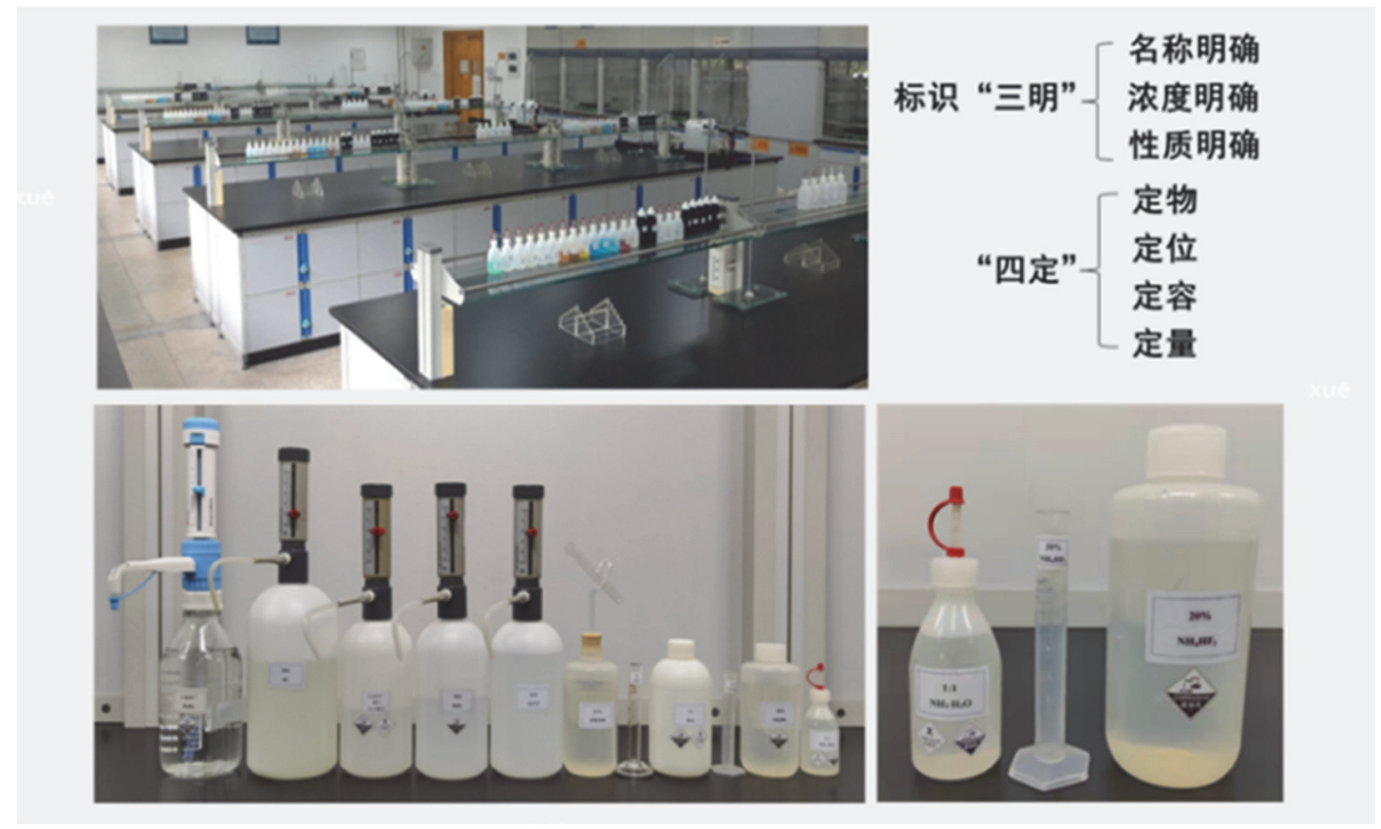

图5 学生实验室化学试剂实行 “标识三明” 和 “四定” 管理示例

\section{3 真诚服务于师生的 “人性化” 的细化管理}

在 “厦大实验中心”, “以人为本, 用心工作” 的服务意识已经渗透于实验室建设与管理的方方 面面, 如在 “厦大实验中心” 设有供师生借用的实验服和护目镜专柜(图6)。以前规定, 学生没有穿 实验服和戴护目镜, 不准进实验室做实验, 常有个别学生忘带实验服或护目镜赶回宿舍去取, 跑回 来满头大汗, 有时在路上急忙中出现安全问题, 有时会迟到等, 影响实验教学……现在, 师生需 要实验服或护目镜时可自助借用, 用完后放回指定地方, 教辅人员对其进行洗涤或清洗后再放回专 柜中, 为师生提供真诚的人性化服务。

另外, 还在指定地方设置了 “失物招领” 专柜, 将学生遗留在实验室里的教材、实验报告、雨 伞等物品收集于其中, 由学生自行领取; 下雨时, 没有带雨伞的同学可以自行从 “失物招领” 专柜 中取用雨伞, 方便时自行归还。对实验室的人性化管理, 就是以人为中心, 把尊重人、爱护人、关 心人作为实验教学服务的出发点。
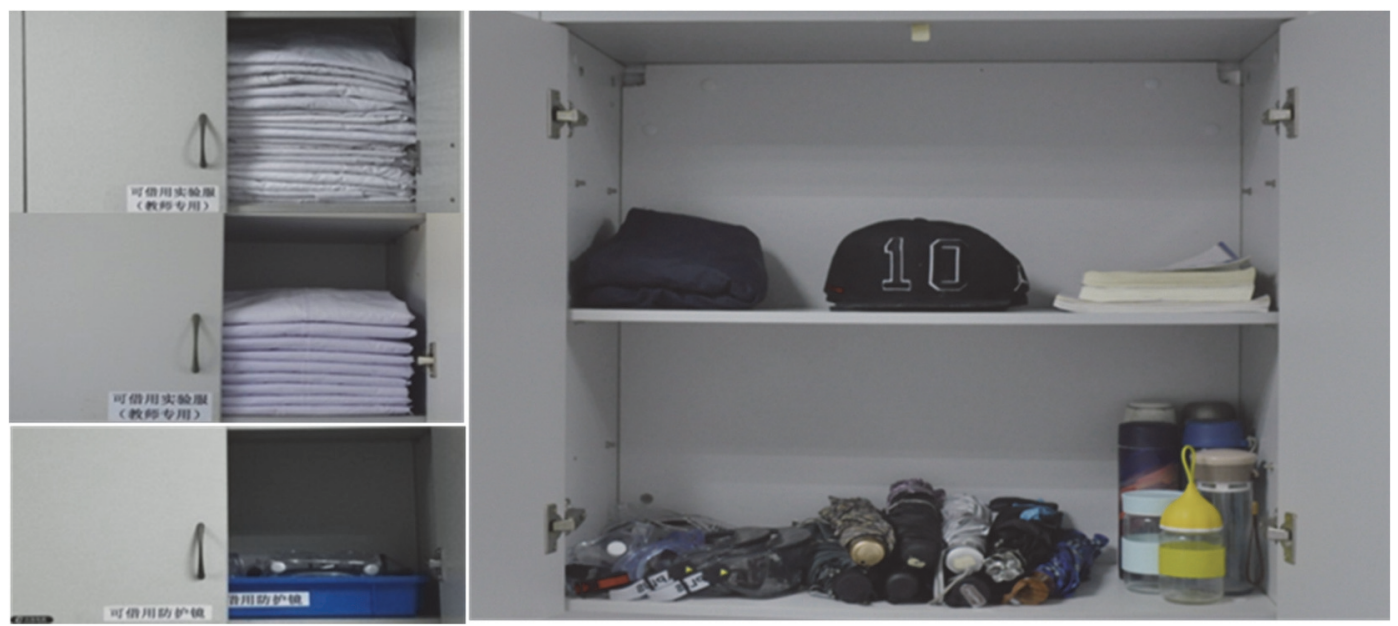

图6 实验服、护目镜及 “失物招领” 专柜 
在 “无机及分析化学” 实验中, 电子天平是常用的精密仪器, 要专门放在 “五防” 的房间中, 但空调气流影响天平的称量, 为了提高学生的实验效率及称量的准确性, 细心的教辅人员在空调排 风口加装挡风板(图4)以减小空调气流对电子天平称量的干扰。

“中心标准” 的建立与实施, 对实验室内的人、事、物等进行有效管理, 不仅很好地解决了实 验室房屋环境以及仪器设备的分类、放置和维护等问题, 使得实验室日常管理水平得以提升; 也形 成了师生相互监督、相互促进的双向良性循环的局面, 有力地发挥了其高效服务于实验教学的专业 功能, 保障学生实验的安全、高效进行, 同时还培养了学生保持实验室安全、整齐、整洁的意识和 责任心。保障实验教学良性运行的 “以学生为中心” 的实验室建设与管理 “中心标准” 真正做到管 理标准化和服务人性化。

\section{4 “厦大实验中心” 的信息化、智能化建设与应用}

上述总结了 “厦大实验中心” 在 “以学生为中心” 的实验室细化建设与管理方面所做的工作。 “厦大实验中心” 在 “以学生为中心” 的实验教学及其管理、实验室建设与管理的信息化、智能化 建设方面也取得了重要进展 ${ }^{[15-17]}$, 其内容概要如图7所示。



图7 实验教学及其管理、实验室建设与管理的信息化、智能化建设内容概要

\section{1 二维码的灵活应用}

扫取二维码获取各种信息的应用已经非常普遍 ${ }^{[19,20]}$ 。文献 ${ }^{[15]}$ 已详细报道了 “厦大实验中心” 将 二维码灵活应用于实验教学及其管理、实验仪器管理和学术报告中的一些具体做法, 其应用示例见 图8。

“动态” 教材、实验教学进度表、仪器清单等 “动态” 内容以二维码的形式给出, 这些内容变 化时, 即将二维码链接的后台内容随时进行更新, 而二维码不变, 即 “一码” 到底, 方便学生随时 扫取和保存。通过二维码获取信息直观、方便、快速, 省去查找麻烦(如扫取图9中二维码即可获取 文章内容), 做到与时俱进地为读者提供人性化服务。

\section{2 “实验开放共享平台” 的构建与应用}

前文 ${ }^{[16]}$ 已详细报道了有关 “厦大实验中心” “智能化实验开放共享平台” 的构建框架、管理系统、 运行过程及流程等。“智能化实验开放共享平台” 具有对 “厦大实验中心” 现有资源的查询功能和应 用预约功能, 具体情况如图7所示。 

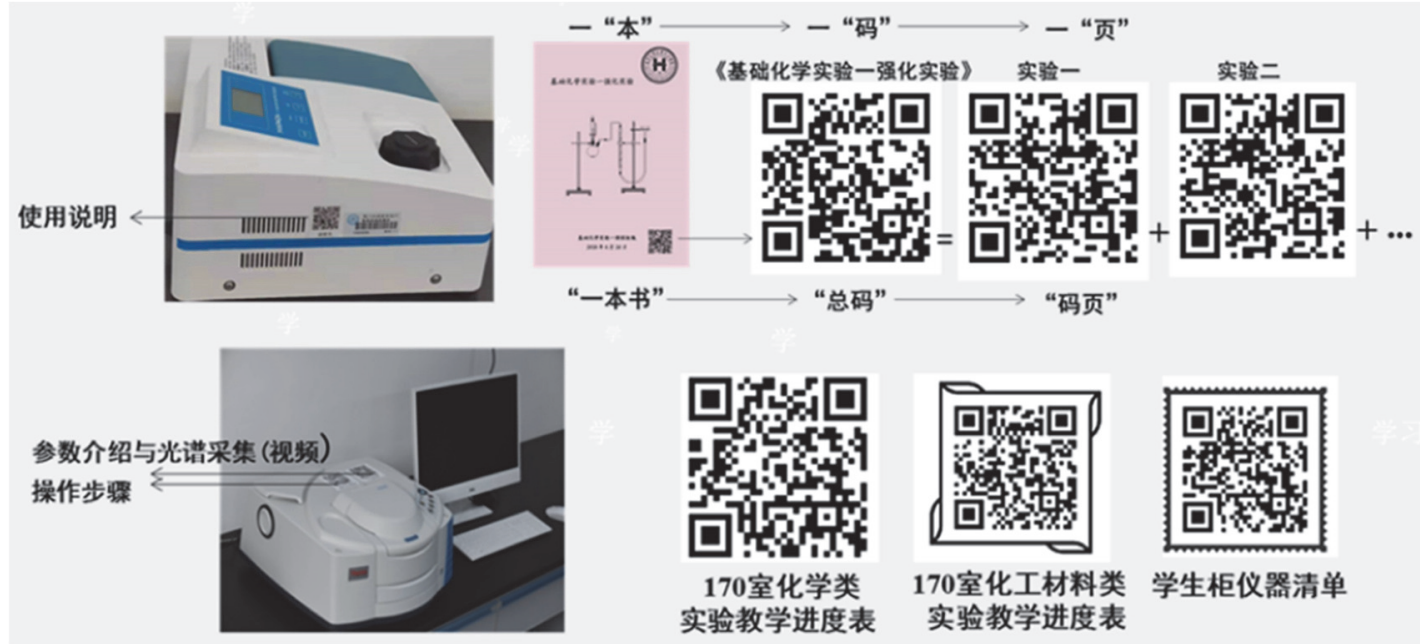

图8 二维码在实验教学及其管理、实验仪器管理等应用示例

有关实验室细化建设和管理方面的具体做法、经验和体会 已以论文形式在 “大学化学” 杂志发表

1. “6S” 在高校基础化学教学实验室细化管理中的灵活应用, 大学化学, 2017, 32 (4), 40-45; 本期亮点文章, 重点推荐给读者

2. 物理化学实验室真空与气路系统的改进, 大学化学, 2018,33 (5), 33-37;

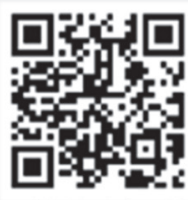

10篇论文汇总

3. 如何精准做好化学教学实验室的建设, 大学化学, 2018, 33 (12),39-45; 本期亮点文章, 重点推葆给读者

4. 科学做好基础化学实验的细化准备, 大学化学, 2019, 34 (2) 37-43; 本期亮点文章, 重点推荐给读者

5. 基础化学教学实验室试剂和仪器的科学细化管理. 大学化学, 2019,34 (5), 57-64

6. 浅谈规范工作流程在有机化学实验室日常管理中的实践, 大学化学, 2020,35(7): 118-122

7. 二维码在实验教学和实验仪器管理以及学术报告中的灵活应用, 大学化学, 2021,36 (2): 1912017

8. 构建智能化实验开放共享平台的探索, 大学化学, 2021, 36 (2): 2003064

9. 射频识别技术结合人脸识别技术用于试剂 “细化” “动态” 管理, 大学化学, 2021,36 (4): 2004091

10. 加强实验教学环境内涵建设 提高环境育人质量, 大学化学, DOI: 10.3866/PKU.DXHX 202104008

图9 《大学化学》杂志发表的有关论文

\section{3 化学试剂 “细化” “动态” 管理系统开发与应用}

现在，在国际大背景下，对化学试剂的管控越来越严格，对易制毒试剂的管控范围越来越广。

为了使高校对化学试剂管理全过程做到完全 “动态” 跟踪记录管理和有效实施, “厦大实验中心” 率 先引入国际上流行的射频识别技术和人脸识别技术, 开发出一套试剂 “细化” “动态” 管理系统。前 文 ${ }^{[17]}$ 已详细介绍了该管理系统的构成、使用流程等。通俗地说, 该系统就是将物、人、时、量自动 关联起来, 即什么人什么时间买了什么试剂、买了多少以及什么时候谁用的、用了多少、剩了多少、 用在哪里等详细流水信息的智能化管理，就是所谓的试剂 “细化” “动态”管理。

有关专家评价 “用射频识别技术结合人脸识别技术用于试剂 “细化” 和 “动态” 管理, 在高校 化学教学实验室管理系统中是一种创新的试剂管理技术。通过应用和实践, 证明该技术在试剂管理 上比现有各种管理方法有较突出的特点和优势, 具有较大的推广价值。”

\section{5 实验室细化建设与管理的示范与辐射效应}

为了让 “厦大实验中心” 的实验室建设与管理成果惠及和辐射全国高校, 主要通过论文、应邀 走出去和迎进来的方式与其他高校专家、同行进行交流。 
在实验室细化建设与管理的实践过程中, 我们也及时对有关如何精准做好实验室的建设和管理 的具体做法、经验和体会进行了初步探索和总结, 并以论文形式在 “ 《大学化学》杂志上发表, 详 细情况如图9所示(用手机扫描图9中的二维码, 可以查看所列论文的详细内容), 也集中展示了 “厦大 实验中心”在实验室细化建设与管理方面所取得的成果。

近几年来, “厦大实验中心” 的多名成员也走出去参加 “全国大学生化学实验邀请赛暨实验教 学研讨会” “全国高校化学实验技术交流会”、福建省的有关实验教学研讨会、四川省 “第三届大 学生化学实验竞赛” 暨实验教学研讨会和第十二届山东省化学实验大赛暨山东省高等院校化学实验 教学与实验室建设研讨会等, 并应邀作大会报告, 通过实例阐述了 “厦大实验中心” 把立德树人的 理念融入到化学实验教学实验室建设与管理体系中, 围绕 “以学生为中心” 的理念, 提升实验环境 育人功能, 强化实验环境内涵建设方面的一些做法和体会, 引起了与会者的强烈认同。

“厦大实验中心” 率先提出的 “安全、规范、高效服务实验教学的实验室细化建设与管理” 理 念, 受到领导、专家、同行的高度认可, 并纷纷表示, “厦大实验中心” 率先提出并实施的高校教 学实验室的管理理念和赋予责任心的细化建设经验值得所有高校学习, 并已在全国高校产生了强烈 的示范辐射效应。

近几年来, “厦大实验中心” 集中或分批接待了清华大学、北京大学、福州大学等100多所高校 1000 余人前来参观学习交流。“厦大实验中心” 在化学教学实验室精准建设与细化管理方面给大家 留下了深刻印象(https://hxsyzx.xmu.edu.cn/)。并与福州大学等高校开展了有关人员的实验教学示范、 新进技术人员培训等, 相关高校教师还通过微信群随时以图片、视频、语音、文字等形式交流有关 实验室建设与管理的片段成果, 以促进共同成长。

另外, “厦大实验中心” 与忻州师范学院、内蒙古民族大学建立了结对帮扶合作关系, 就化学 实验教学、实验室建设与管理等方面签订了结对帮扶合作框架协议, 并多举措组织开展了实质性的 帮扶合作活动, 如把 “厦大实验中心” 的有关成果直接应用于忻州师范学院的化学实验教学以及实 验室建设与管理中, 并取得了良好效果(http://chem.xztu.edu.cn/info/1012/1233.htm), 做到了精准帮扶。 厦门大学化学国家级实验教学示范中心也是全国65个化学化工国家级实验教学示范中心中唯一一个 与中西部高校建立帮扶合作关系的示范中心, 这与目前教育部开展的全国虚拟教研室的建设理念非 常契合, 对提高中西部高校的实验教学水平具有重要作用。

\section{6 结语}

安全、规范、高效服务于实验教学的实验室建设与管理是实验教学改革和环境育人的重要基础。 本文在引导读者认识化学实验教学实验室建设与管理的重要性和必要性的基础上, 概括总结了 “厦 大实验中心” 近几年来在实验室细化建设与管理方面所建立和实践的 “以学生为中心” 的 “科学化” 细化管理和 “人性化” 细化管理 “中心标准”, 以及在实验教学及其管理、实验室建设与管理的信息 化、智能化建设方面所取得的重要进展。安全、规范、高效、美观、舒适是有关学生、专家、同行 对 “厦大实验中心” 的真实感受。通过多方交流, 增强了“厦大实验中心” 的示范和辐射作用。

“厦大实验中心”也在不断探讨实验室建设与管理发展的新趋势, 不断积极创新理念和管理模 式, 以求在实验室建设与管理方面做得更好。

\section{参 考 文 献}

[1] 任艳平, 董志强, 阮婵姿. 大学化学, 2015, 30 (2), 22.

[2] 董志强, 任艳平. 大学化学, 2016, 31 (11), 51 .

[3] 任艳平. 大学化学, 2017, 32 (1), 15.

[4] 任艳平, 吕银云, 董志强. 大学化学, 2018, 33 (9), 55. 
[5] 董志强, 吕银云, 任艳平. 大学化学, 2018, 33 (9), 88 .

[6] 任艳平, 吕银云, 郑啸, 林敏, 夏文生. 大学化学, 2019, 34 (10), 95.

[7] 吕银云, 阮婵姿, 张春艳, 潘莣, 许振玲, 任艳平. 大学化学, 2020, $35(9), 89$.

[8] 王翊如, 邓顺柳, 吕银云, 任艳平. 大学化学, 2021, 36 (4), 2011014.

[9] 欧阳小清, 张春艳, 潘荵, 阮婵姿, 吕银云, 翁玉华, 董志强, 彭淑女, 李华敏, 许振玲, 等. 大学化学, 2021, 36 (3), 2006069.

[10] 任艳平, 王翊如，曹晓宇，吕银云，翁玉华，邓顺柳. 大学化学, 2021, 36 (12), 2103034

[11] 张春艳, 翁玉华, 董志强, 欧阳小清, 阮婵姿, 潘荵, 许振玲, 颜长明, 任艳平. 大学化学, 2017, 32 (4), 40.

[12] 翁玉华, 颜长明, 张春艳, 阮永红, 张来英, 彭淑女, 方雪明, 任艳平. 大学化学, 2018, 33 (12), 39.

[13] 张春艳, 吕银云, 潘荵, 翁玉华, 阮婵姿, 欧阳小清, 董志强, 许振玲, 颜长明, 任艳平. 大学化学, 2019, 34 (2), 37.

[14] 欧阳小清, 董志强, 阮婵姿, 潘荵, 吕银云, 张春艳, 翁玉华, 许振玲, 颜长明, 任艳平. 大学化学, 2019, 34 (5), 64.

[15] 吕银云, 翁玉华, 潘荵, 张春艳, 董志强, 欧阳小清, 阮婵姿, 许振玲, 彭淑女, 任艳平. 大学化学, 2021, 36 (2), 1912017.

[16] 吕银云, 潘荵, 翁玉华, 阮永红, 吴平平, 张来英, 彭淑女, 陈玉清, 张春艳, 董志强, 等. 大学化学, 2021, 36 (2), 2003064.

[17] 翁玉华, 潘荵, 许振玲, 颜长明, 欧阳小清, 董志强, 张春艳, 阮婵姿, 吕银云, 任艳平. 大学化学, 2021, 36 (4), 2004091.

[18] 翁玉华, 许振玲, 潘荵, 张春艳, 阮婵姿, 吕银云, 董志强, 方雪明, 张来英, 彭淑女, 等. 大学化学, 2022, 37 (1), 2104008 .

[19] 二维码的具体应用. [2021-09-14]. https://wenda.so.com/q/1387164235068334? src=140\&q=

[20] 闰晓义, 马强, 谷月, 魏士刚, 张志权, 郭玉鹏. 大学化学, 2022, 37 (2), 2105037. 